\title{
Designs of Compact, Planar, Wideband, Monopole Filtennas with Near-Field Resonant Parasitic Elements
}

\author{
Ming-Chun Tang ${ }^{1}$, Yang Wang ${ }^{1}$, Ying Chen ${ }^{1}$, and Richard W. Ziolkowski ${ }^{2,3}$ \\ ${ }^{1}$ College of Communication Engineering, Chongqing University, Chongqing, China, tangmingchu@cqu.edu.cn \\ ${ }^{2}$ University of Technology Sydney, Global Big Data Technologies Centre, Ultimo NSW 2007, Australia, \\ ${ }^{3}$ Department of Electrical and Computer Engineering, University of Arizona, Tucson, AZ 85721, USA, \\ Richard.Ziolkowski@uts.edu.au
}

\begin{abstract}
Two planar efficient wideband, electrically small monopole filtennas are presented. The first one directly evolves from a common planar capacitively loaded loop (CLL)-based filter possessing a flat realized gain response within the operational band and good band-edge selectivity. The second filtenna consists of a driven element augmented with a CLL structure and with slots etched into its ground plane. It expands the fractional impedance bandwidth of the first case from $6.28 \%$ up to $7.9 \%$. It also has a gain response that remains flat over its operational bandwidth and even higher band-edge selectivity. Both filtennas are electrically small: $k a<1$. The experimental results, which are in good agreement with their simulated values, demonstrate that both filtennas exhibit excellent impedance matching, high radiation efficiency, flat gain response, and steep skirts at both band edges. Moreover, they produce monopole radiation patterns that are uniform and nearly omnidirectional in their $\mathbf{H}$-planes.
\end{abstract}

Index Terms-Bandwidth, efficient, electrically small antenna, filtenna, near-field resonant parasitic (NFRP) element, planar antenna.

\section{Introduction}

Filtennas are co-designed antennas that combine a radiating element and a filter into a single module. Because of its self-contained filtering function, a filtenna has three advantages over common antennas when a signal is received. First, it reduces the interconnection losses, occurring when a standard receiving antenna is connected to a filter in the processing layer. Second, it suppresses unwanted signals out of the operational band. Third, from a practical point of view, it facilitates a more compact and lower-cost RF front-end system. As a consequence, a variety of filtennas have been developed. Examples include filtering horn antennas [1]-[3], substrate integrated waveguide filtennas [4], [5], planar filtering ultra-wide band (UWB) antennas [6], [7], filtering microstrip patch antennas [8]-[10], filtering slot antennas [11], and filtering quasi-Yagi antennas [12]. Efforts to miniaturize and compactify filtennas have employed composite right-/lefthanded transmission lines (CRLH-TLs) [13], and multiple electrically small resonators in series [14], [15]. Unfortunately, the relatively large radiation patch/long meander-line strip above the ground required in those CRLH-TL-based filtenna designs prohibits further miniaturization [13]. Similarly, a multiresonator filtenna makes use of multipole filter design principles, exploiting the presence of more transmission poles to achieve broader operational bandwidths. This inescapably increases their electrical sizes [14], [15], which are deemed to be not small, i.e., $k a>1$ (where $a$ is the radius of the smallest sphere that completely encloses the entire antenna at the lowest operational frequency, $f_{L}$, and $k=2 \pi / \lambda_{0}=2 \pi f_{L} / c$ is the corresponding free space wavenumber) [13]-[15]. In this paper, we report two electrically small, efficient planar monopole filtennas based on capacitively loaded loop (CLL) resonators. Taking advantage of the characteristics of filters that are based on a pair of electrically coupled CLL resonators, we designed, fabricated, and measured the first filtenna. As discussed in Section II, the experimental results demonstrate that this electrically small system had a $6.27 \%$ fractional impedance bandwidth, high out-of-band rejection, and stable omnidirectional radiation patterns. An additional CLL structure, as a near-field resonant parasitic (NFRP) element, is then integrated systematically into the system to achieve a wider operational bandwidth. The resulting filtenna has a $7.9 \%$ fractional bandwidth, together with a flat gain response, stable omnidirectional radiation patterns, and high out-ofband rejection characteristics.

\section{Electrically Small Filtenna Design}

A filtenna having a second-order filter was codesigned and optimized. It is shown in Fig. 1. Fig. 1(a) indicates that one CLL element acts as the directly driven element. A fan-shaped radiator with no ground plane on the back side of it acts as a NFRP element in the presence of the monopole (CLL-based) antenna [16]. The choice of this special fan-shaped radiator establishes an even smoother impedance transition over the desired wider bandwidth. Simply starting with the resonance frequencies near to each other facilitates a straight forward numerical approach to optimized and finalize the actual antenna design. Note that the fan-shaped part of the NFRP element is placed on the opposite side of the feed port. This arrangement facilitates the creation of dual transmission zeros on the two edges of the passband. This arrangement enhances the out-of-band rejection level. 


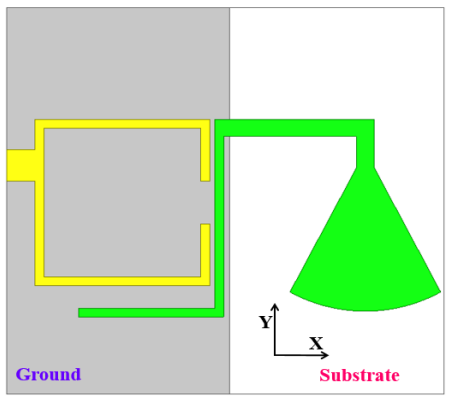

(a)

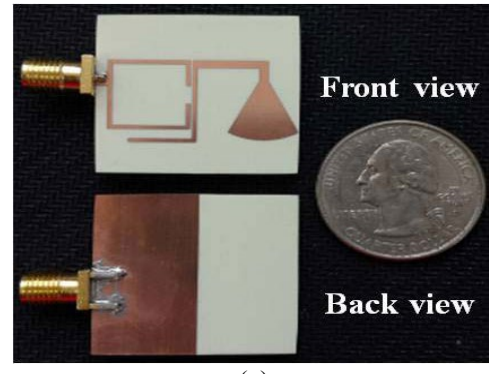

(c)

Fig. 1. First electrically small filtenna. (a) Top and (b) side views of the HFSS simulation model. (c) Front and back views of the fabricated prototype.

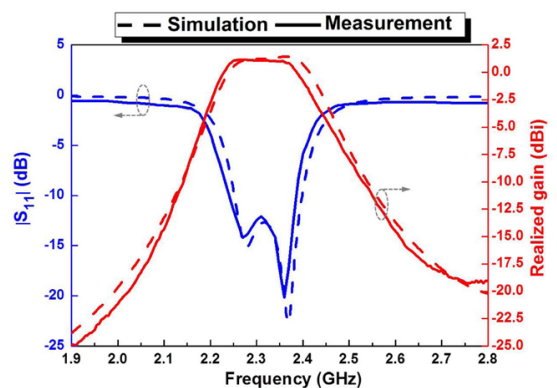

Fig. 2. Measured and simulated $\left|S_{11}\right|$ and realized gain values of the first filtenna as functions of the source frequency.

The measured and simulated $\left|S_{11}\right|$ values are presented in Fig. 2. The measured values confirmed that the filtenna had a - 10-dB impedance bandwidth from 2.24 to $2.385 \mathrm{GHz}$ (6.27\% fractional impedance bandwidth) in good agreement with the simulated values $2.252-2.398 \mathrm{GHz}(6.28 \%$ fractional impedance bandwidth). The electrical size of the measured prototype is $k a \sim 0.93$, while its simulated value was $\sim 0.935$. Fig. 2 demonstrates that the prototype filtenna has a flat realized gain response within its passband. The measured (simulated) peak value was $1.15(1.41) \mathrm{dBi}$. The simulated radiation efficiency was higher than $80.93 \%$ throughout the operational band. This realized Filtenna prototype clearly has very good band-edge selectivity and stopband suppression.

\section{Filtenna with Enhanced Bandwidth}

For many applications it is desirable to have an even wider bandwidth. Consequently, the second design shown in Fig. 3 was considered. In order to improve the flatness of the transmission performance within the passband while maintaining its wideband operation and steep skirts, a third resonator was introduced without increasing the total overall dimension of the filtenna.

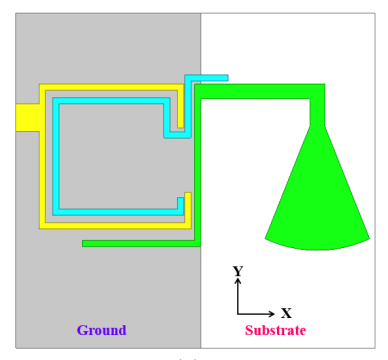

(a)

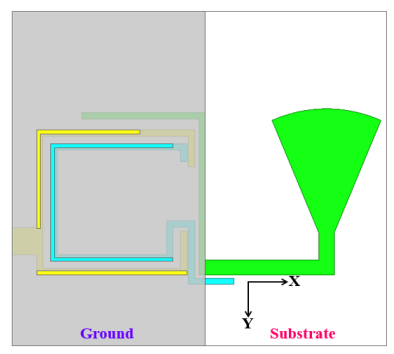

(b)

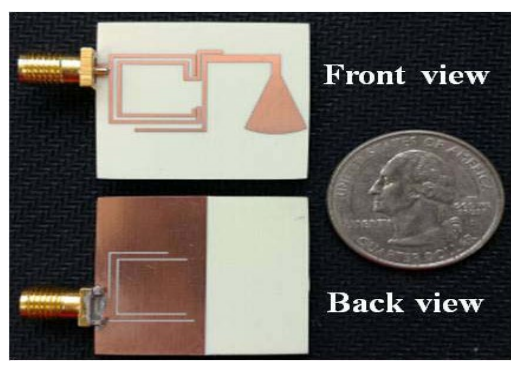

(c)

Fig. 3. Enhanced bandwidth filtenna with slots in its ground strip. (a) Top and (b) back views of the HFSS simulation model. (c) Front and back views of the fabricated prototype.

The third resonator is an additional CLL element, shown in blue in Fig. 3(a). Its gap position coincides with the driven CLL element, and it has an arm included to facilitate its coupling to the NFRP element. This collocated arrangement of the two CLLs provides a means to control the mutual coupling, further expanding the bandwidth without increasing the total overall dimensions of the filtenna. Three slots were etched in the ground strip directly beneath the two CLL elements to achieve a smoother realized gain curve. The length of the additional CLL element is set nearly equal to the driven CLL's size to make their resonance frequencies close to one another.

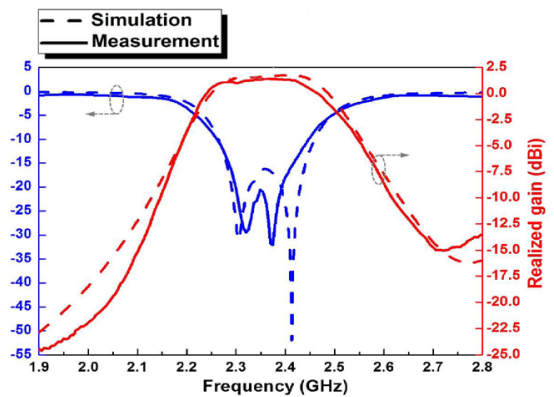

Fig. 4. Measured and simulated $\left|S_{11}\right|$ and realized gain values as functions of the source frequency for the enhanced bandwidth filtenna with slots in its ground strip.

The simulated and measured $\left|S_{11}\right|$ and realized gain values of the second filtenna with the ground strip slots are given in 
Fig. 4. The simulated (measured) realized gain values indicate that the peak realized gain was increased from 1.659 to 1.75 $\mathrm{dBi}$ in simulation. It was measured to be $1.376 \mathrm{dBi}$, indicating that more losses were encountered than expected in the fabricated prototype. From the simulated $\left|S_{11}\right|$ values presented in Fig. 4, the impedance bandwidth was determined to be from 2.264 to $2.46 \mathrm{GHz}(8.3 \%$ fractional bandwidth, i.e., a $32.2 \%$ enhancement) and was from 2.261 to $2.447 \mathrm{GHz}(7.9 \%$ fractional bandwidth, i.e., a $26 \%$ enhancement) in the measurement. Similarly, the simulated $k a \sim 0.94$ and measured $k a \sim 0.938$ values verify that the filtenna is electrically small. Furthermore, the simulated radiation efficiency across the entire operational bandwidth is higher than $82.87 \%$. Again, very good agreement between the simulated and measured performance characteristics was obtained.

\section{Conclusion}

We have explained the basic design principles to obtain two planar electrically small wideband filtennas. The first design evolved from a two-pole filter consisting of a pair of CLL elements. The resulting two-pole filtenna was achieved with a single driven CLL element and an appropriate fanshaped NFRP element that was the primary radiator. It was demonstrated that this simple filtenna possesses a flat realized gain response within its operational band and has good bandedge selectivity. To increase the bandwidth while maintaining its steep skirts at the band edges while maintaining its electrically small size, a second CLL element was integrated into the system. The majority of this second NFPR element was located inside the region circumscribed by the driven CLL element. Several of its segments were external to that region to achieve the desired resonant length and requisite capacitive coupling levels. By introducing several slots into the ground strip beneath the CLL elements, a smother realized gain curve was achieved. Prototypes of both filtennas were fabricated and measured. The simulation and measured results matched very well.

\section{References}

[1] G. Q. Luo et al., "Filtenna consisting of horn antenna and substrate integrated waveguide cavity FSS," IEEE Trans. Antennas Propag., vol. 55, no. 1, pp. 4933-4939, Jan. 2007.

[2] M. Barbuto, F. Trotta, F. Bilotti, and A. Toscano, "Horn antennas with integrated notch filters," IEEE Trans. Antennas Propag., vol. 63, no. 2, pp. 781-785, Feb. 2015.

[3] M. Barbuto, F. Trotta, F. Bilotti, and A. Toscano, "Varying the operation bandwidth of metamaterial-inspired filtering modules for horn antennas," Prog. Electromagn. Res. C, vol. 58, pp. 61-68, 2015.

[4] C. Yu, W. Hong, Z. Kuai, and H. Wang, "Ku-band linearly polarized omnidirectional planar filtenna," IEEE Antennas Wireless Propag. Lett., vol. 11, pp. 310-313, 2012.

[5] O. A. Nova, J. C. Bohórquez, N. M. Peña, G. E. Bridges, L. Shafai, and C. Shafai, "Filter-antenna module using substrate integrated waveguide cavities," IEEE Antennas Wireless Propag. Lett., vol. 10, pp. 59-62, 2011.

[6] S. W. Wong, T. G. Huang, C. X. Mao, Z. N. Chen, and Q. X. Chu, "Planar filtering ultra-wideband (UWB) antenna with shorting pins," IEEE Trans. Antennas Propag., vol. 61, no. 2, pp. 948-953, Feb. 2013.

[7] M.-C. Tang, T. Shi, and R. W. Ziolkowski, "Planar ultrawideband antennas with improved realized gain performance," IEEE Trans. Antennas Propag., vol. 64, no. 1, pp. 61-69, Jan. 2016.

[8] J. Zuo, X. Chen, G. Han, L. Li, and W. Zhang, "An integrated approach to RF antenna-filter co-design," IEEE Antennas Wireless Propag. Lett., vol. 8, pp. 141-144, 2009.

[9] C. K. Lin and S. J. Chung, "A compact filtering microstrip antenna with quasi-elliptic broadside antenna gain response," IEEE Antennas Wireless Propag. Lett., vol. 10, pp. 381-384, 2011.

[10] Z. H. Jiang and D. H. Werner, "A compact, wideband circularly polarized co-designed filtering antenna and its application for wearable devices with low SAR," IEEE Trans. Antennas Propag., vol. 63, no. 9, pp. 3808-3818, Sep. 2015.

[11] X. Chen, F. Zhao, L. Yan, and W. Zhang, "A compact filtering antenna with flat gain response within the passband," IEEE Antennas Wireless Propag. Lett., vol. 12, pp. 857-860, 2013.

[12] J. Shi et al., "A compact differential filtering quasi-Yagi antenna with high frequency selectivity and low cross-polarization levels," IEEE Antennas Wireless Propag. Lett., vol. 14, pp. 1573-1576, 2015.

[13] L. Chen and Y.-L. Luo, "Compact filtering antenna using CRLH resonator and defected ground structure," Electron. Lett., vol. 50, no. 21, pp. 1496-1498, Oct. 2014.

[14] P.-C. Wu, L. Chen, and Y.-L. Luo, "Miniaturised wideband filtering antenna by employing CRLH-TL and simplified feeding structure," Electron. Lett., vol. 51, no. 7, pp. 548-550, Apr. 2014.

[15] W.-J. Wu, Y.-Z. Yin, S.-L. Zuo, Z.-Y. Zhang, and J.-J. Xie, "A new compact filter-antenna for modern wireless communication systems," IEEE Antennas Wireless Propag. Lett., vol. 10, pp. 1131-1134, 2011.

[16] R. W. Ziolkowski, P. Jin, and C.-C. Lin, "Metamaterial-inspired engineering of antennas," Proc. IEEE, vol. 99, no. 10, pp. 1720-1731, Oct. 2011. 